\title{
Práticas Integrativas e Complementares no tratamento de crianças com câncer: uma
}

\section{revisão integrativa da literatura}

\author{
Integrative and Complementary Practices in treating children with cancer: an integrative literature \\ review
}

Prácticas Integrativas y Complementarias en el tratamiento de niños con cáncer: una revisión integrativa de la literatura

Recebido: 06/10/2021 | Revisado: 11/10/2021 | Aceito: 18/10/2021 | Publicado: 20/10/2021

\author{
Lynna Stefany Furtado Morais \\ ORCID: https://orcid.org/0000-0002-5611-2736 \\ Universidade Federal do Triângulo Mineiro, Brasil \\ E-mail: lynnastefany.morais@gmail.com \\ José Humberto Alves \\ ORCID: https://orcid.org/0000-0003-0573-230X \\ Universidade de Uberaba, Brasil \\ E-mail: jhfisioterapeuta@gmail.com \\ Andrea Ruzzi Pereira \\ ORCID: https://orcid.org/0000-0001-6014-0468 \\ Universidade Federal do Triângulo Mineiro, Brasil \\ E-mail: andrea.pereira@uftm.edu.br
}

\begin{abstract}
Resumo
Introdução: As Práticas Integrativas e Complementares em Saúde envolvem abordagens que buscam a prevenção de agravos e a recuperação da saúde por meio de mecanismos tecnológicos eficazes e seguros, promovem a escuta acolhedora, o vínculo terapêutico e a integração entre ser humano, meio ambiente e sociedade. Objetivo: Investigar o que foi publicado na literatura nacional e internacional sobre a utilização das Práticas Integrativas e Complementares no tratamento de crianças com câncer. Metodologia: Revisão de literatura do tipo integrativa, utilizando as bases de dados: MEDLINE/PubMed, Biblioteca da Literatura Latino-Americana e do Caribe em Ciências da Saúde (LILACS), Scopus e Web Of Science. Resultados: Foram analisados nove artigos da síntese qualitativa, que abordavam as práticas integrativas e complementares utilizadas na atenção a crianças com câncer. Os tipos de práticas encontradas foram Reike, Apiterapia, Arteterapia, Plantas medicinais (fitoterapia) e Terapia comunitária integrativa. Conclusão: Conclui-se que os estudos apresentados apontam uma contribuição significativa das práticas integrativas associadas ao tratamento do câncer infantil. No entanto, há a necessidade de replicar esses achados em outros estudos mais aprofundados para desenvolver uma melhor compreensão acerca do tema, além de estudos relatando a aplicabilidade de certas práticas para atuação do profissional que pretende trabalhar com a temática.
\end{abstract}

Palavras-chave: Terapias complementares; Neoplasias; Criança.

\begin{abstract}
Introduction: Integrative and Complementary Practices involve approaches that seek to prevent health problems and recover health through effective and safe technological mechanisms, promote warm listening, therapeutic bonding and integration between human beings, the environment and society. Objective: To investigate what has been published in the national and international literature on the use of Integrative and Complementary Practices in the treatment of children with cancer. Methodology: Integrative literature review, using the following databases: MEDLINE/PubMed, Library of Latin American and Caribbean Literature in Health Sciences (LILACS), Scopus and Web of Science. Results: Nine qualitative synthesis articles were analyzed, which addressed the integrative and complementary practices used in the care of children with cancer. The types of practices found were Reike, Apitherapy, Art Therapy, Medicinal Plants (phytotherapy) and Integrative Community Therapy. Conclusion: It is concluded that all studies presented point to a significant contribution of integrative practices associated with the treatment of childhood cancer. However, there is a need to replicate these findings in other more in-depth studies to develop a more sophisticated understanding of the topic, in addition to studies reporting the applicability of certain practices to the work of professionals who intend to work with the theme.
\end{abstract}

Keywords: Complementary therapies; Neoplasms; Child.

\section{Resumen}

Introducción: Las Prácticas Integrativas y Complementarias en Salud involucran enfoques que buscan prevenir problemas de salud y recuperar la salud a través de mecanismos tecnológicos efectivos y seguros, promover la 
escucha acogedora, la vinculación terapéutica y la integración entre los seres humanos, el medio ambiente y la sociedad. Objetivo: Investigar lo publicado en la literatura nacional e internacional sobre el uso de Prácticas Integrativas y Complementarias en el tratamiento de niños con cáncer. Metodología: Revisión integrativa de la literatura, utilizando las siguientes bases de datos: MEDLINE / PubMed, Biblioteca de Literatura Latinoamericana y del Caribe en Ciencias de la Salud (LILACS), Scopus y Web Of Science. Resultados: Se analizaron nueve artículos de síntesis cualitativa, que abordaron las prácticas integradoras y complementarias utilizadas en la atención de niños con cáncer. Los tipos de prácticas encontradas fueron Reike, Apiterapia, Arteterapia, Plantas Medicinales (fitoterapia) y Terapia Comunitaria Integrativa. Conclusión: Se concluye que todos los estudios presentados apuntan a una contribución significativa de las prácticas integradoras asociadas al tratamiento del cáncer infantil. Sin embargo, existe la necesidad de replicar estos hallazgos en otros estudios más profundos para desarrollar una comprensión más sofisticada del tema, además de estudios que reporten la aplicabilidad de ciertas prácticas al trabajo de los profesionales que pretenden trabajar con el tema.

Palabras clave: Terapias complementarias; Neoplasias; Niño.

\section{Introdução}

As Práticas Integrativas e Complementares em Saúde (PICS) envolvem abordagens que buscam a prevenção de agravos e a recuperação da saúde por meio de mecanismos tecnológicos eficazes e seguros, promovendo a escuta acolhedora, o vínculo terapêutico e a integração entre ser humano, meio ambiente e sociedade (Brasil, 2015).

No Brasil, existe uma Política que versa sobre as PICS, a Política de Práticas Integrativas e Complementares no Sistema Único de Saúde (PNPIC), instituída no ano de 2006 pelo Ministério da Saúde, garantindo a ampliação do acesso a serviços de saúde antes restritos a prática de cunho privado (Silva, Souza, Cabral et al., 2020). A partir da PNPIC, as PICS foram amplamente difundidas em todos os serviços de saúde do país, tendo destaque para a acupuntura, a homeopatia, a fitoterapia, o termalismo, crenoterapia e a medicina antroposófica (Miotto, 2018).

Em março de 2017, foi lançada a portaria 849/2017 em âmbito nacional, que incluiu 14 novas práticas de saúde: arteterapia, biodança, reiki, shantala, medicina ayurveda, dança circular, meditação, musicoterapia, naturopatia, osteopatia, quiropraxia, reflexoterapia, terapia comunitária e yoga (Habimorad, Catarucci, Bruno et al., 2020). A Portaria $\mathrm{n}^{\circ} 702 / 2018$, no que lhe concerne, integrou mais 10 práticas ao Sistema Único de Saúde (SUS), a apiterapia, aromaterapia, bioenergética, constelação familiar, cromoterapia, geoterapia, hipnoterapia, imposição de mãos, ozonoterapia e terapia de florais (Dacal \& Silva, 2018).

A visão ampliada do processo saúde doença e o cuidado humanizado preconizado pelo SUS, tem as PICS como uma alternativa comum no tratamento de pacientes com diversas enfermidades, oncológicos, bem como, os resultados existentes que comprovam a sua eficácia na prevenção e na intervenção em saúde, esses pacientes estão insatisfeitos com as técnicas convencionais e buscam outros métodos que não sejam baseados no tradicional modelo biomédico (Alves, Nascimento, Assis et al., 2015).

Segundo informações do Departamento Nacional da Atenção Básica, Ministério da Saúde (MS), nos níveis de atenção à saúde as PICS estão distribuídas, em sua maioria, na Atenção Básica. Ainda, dados demonstram que mais de 50\% dos estados brasileiros apresentam as PICS em sua Rede de Atenção à Saúde (Tesser, Souza \& Nascimento, 2018).

O câncer pode ser definido como uma neoplasia maligna em que há um crescimento celular rápido e anormal, apesar de existirem muitas técnicas terapêuticas, ainda é uma doença que causa sentimentos de desesperança e sofrimento (Marcião, Sousa, Lima et al., 2021). Nesse sentido, já existem comprovações de que as PICS podem amenizar os efeitos colaterais do tratamento oncológico (Menin, 2020).

Em consonância com dados do Instituto Nacional do Câncer José Alencar Gomes da Silva (INCA), o número de crianças e adolescentes com câncer por ano ultrapassa os dez mil casos em todo o Brasil. Ainda que haja sobrevivência estimada de $70 \%$ da população infantil acometida por essa doença, os tratamentos convencionais são longos e intensos, levando a família a buscar outras alternativas terapêuticas (Caires, Andrade, Amaral et al., 2014). 
De modo geral, com o crescente interesse no uso das medicinas alternativas e complementares, a sua utilização associada aos métodos tradicionais tem efeitos positivos no tratamento do câncer infantil (Costa, Silva, Feitosa et al, 2020). Dito isso, esta pesquisa buscou investigar o que foi publicado na literatura nacional e internacional sobre a utilização das Práticas Integrativas e Complementares no tratamento de crianças com câncer.

Justifica-se a necessidade dessa revisão de modo a identificar lacunas e atualizar profissionais da área sobre o que foi produzido sobre essa abordagem. Desse modo, espera-se que a revisão seja útil para que os profissionais de saúde consigam aliviar o sofrimento infantil no tratamento do câncer, urgindo a necessidade de adaptação das terapêuticas atuais. Além disso, é importante que os responsáveis pela criança tenham conhecimento das possibilidades de tratamento com as PICS, instigando novas estratégias de minimização dos sintomas e busca da cura.

\section{Metodologia}

Trata-se de uma revisão de literatura do tipo integrativa, que tem como definição ser a mais ampla abordagem metodológica referente às revisões, permitindo a inclusão de diferentes estudos, experimentais e não-experimentais, para uma ampla concepção acerca do objeto de estudo a ser analisada. Por meio da revisão integrativa é possível realizar uma extração de conhecimentos e a incorporação da aplicabilidade de resultados de estudos significativos na prática (Souza, Silva \& Carvalho, 2010).

A pesquisa foi realizada em fevereiro de 2021 e seguiu sete fases: elaboração da pergunta norteadora; definição dos critérios de inclusão e exclusão dos artigos encontrados; busca ou amostragem na literatura; extração dos dados dos artigos selecionados; análise crítica dos estudos incluídos; discussão dos resultados e apresentação da revisão integrativa (Sales, Santos, Barbosa et al., 2020).

Definiu-se como pergunta norteadora: "Quais as práticas integrativas utilizadas como complemento no tratamento do câncer em crianças?"

Foram analisados artigos presentes nas seguintes bases de dados: MEDLINE/PubMed, Biblioteca da Literatura Latino-Americana e do Caribe em Ciências da Saúde (LILACS), SciVerse Scopus e Web of Science, que foram escolhidas por serem as principais fontes de publicações da área da saúde. Foram utilizados os seguintes descritores controlados pelo DECS e MESH: Criança (C), Neoplasias (N) e Práticas Integrativas e Complementares (PICS), nas línguas portuguesa, inglesa e espanhola, tendo sido realizados os seguintes cruzamentos entre os descritores: C/N, C/PICS, N/PICS, C/N/PICS.

Os estudos foram selecionados e revisados por dois revisores independentes nas bases de dados por meio das diversas combinações dos descritores: 1- Child AND Complementary Therapies AND Neoplasms; 2- Criança AND Neoplasias AND Terapias Complementares; 3- Niño AND Neoplasias AND Terapias Complementarias. Para composição de cada bloco individual, o descritor principal e seus respectivos termos para a associação dos blocos se utilizou o booleano "AND". Desta busca foram selecionados os artigos para leitura na íntegra que preencheram os critérios de inclusão e exclusão para esta revisão.

Foram considerados os seguintes critérios de inclusão: textos em português, inglês e espanhol, disponíveis na íntegra e gratuitamente nas referidas bases de dados; publicados entre janeiro de 2016 a janeiro de 2021 que apresentassem a relação entre as Práticas Integrativas e Complementares e o tratamento de crianças com câncer. Foram excluídos os artigos em forma de apostilas, cartas e editoriais, artigos de revisão de literatura e metanálise, tendo como foco principal as publicações em periódicos científicos revisadas por pares.

As buscas realizadas pelos autores utilizaram o instrumento de extração de dados para revisão integrativa, adaptado por Ursi e Gavão (2006), o qual contempla as seguintes informações: número do artigo, dados do artigo (título, ano da publicação, idioma, autores, título do periódico, local do estudo) objetivos, método, resultado, recomendações, identificação de 
limites e vieses.

Ainda em relação a coleta de dados, o primeiro autor coletou as informações necessárias para a seleção dos artigos. O segundo autor averiguou todas as informações coletadas para confirmar a precisão. Qualquer desacordo em qualquer fase foi resolvido por reunião e acordo mútuo entre os revisores e o terceiro autor participou de todo o processo envolvido nesta revisão.

A análise dos dados obedeceu às seguintes fases: Identificação dos artigos nas bases de dados, leitura e seleção de artigos pelo título, leitura dos resumos de todos os artigos encontrados, de acordo com os critérios de inclusão e exclusão, leitura na íntegra dos artigos da amostra parcial, exploração dos artigos, codificação dos conteúdos emergentes e relevantes, categorização baseada na incidência do conteúdo e nas características dos estudos, conforme proposto por Bardin (2009).

\section{Resultados}

A forma de seleção dos estudos é apresentada pelo Fluxograma da Figura 1, a seguir. Nele está descrito como ocorreu o processo de inclusão e exclusão dos estudos no decorrer das fases. Foram incluídos nove artigos, segundo os critérios de elegibilidade para esta revisão, conforme mostra a Figura 1.

Figura 1. Fluxograma e critérios de seleção e inclusão dos artigos.
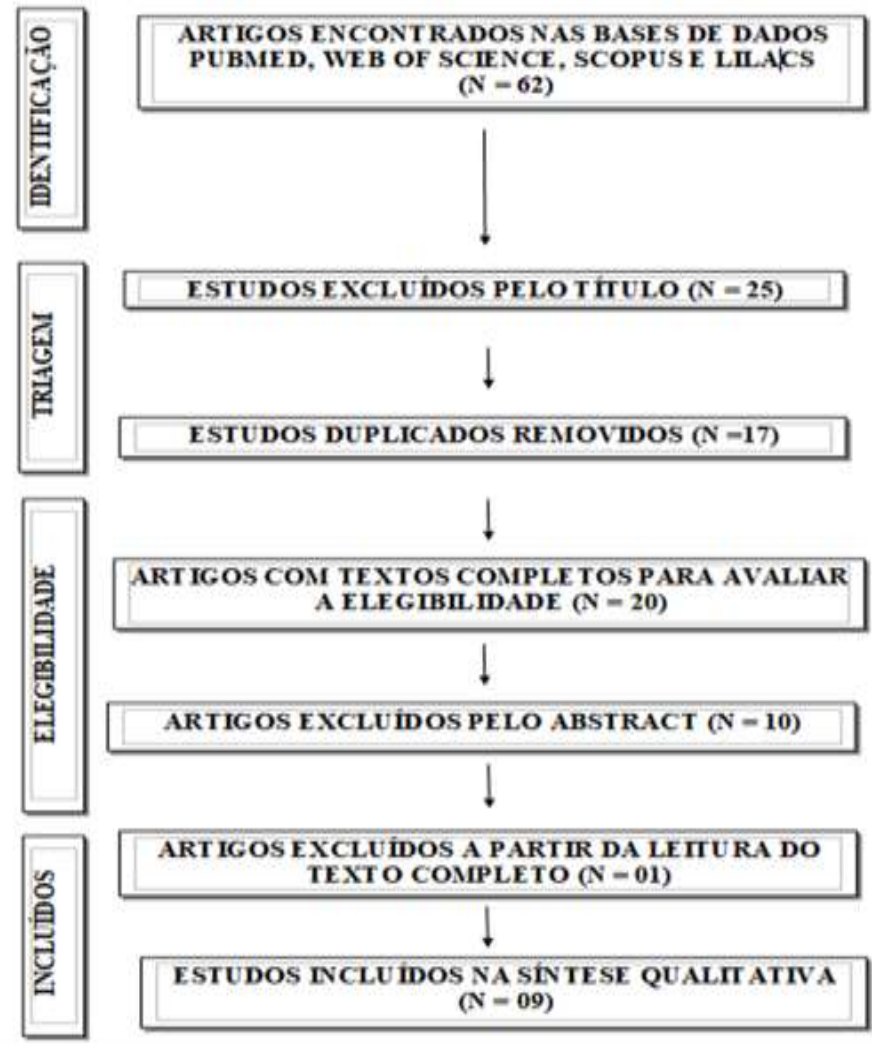

Fonte: Autores (2021).

Observa-se na Figura que, embora tenham sido encontrados 62 artigos e sido elegíveis apenas nove, a literatura recente acerca da utilização das PICS com crianças com câncer ainda é incipiente, considerando-se que foram pesquisados artigos em bases de dados de relevância e em três línguas de maior volume de publicação.

O presente estudo buscou investigar o que foi publicado na literatura nacional e internacional sobre a utilização das Práticas Integrativas e Complementares no tratamento de crianças com câncer. Foram analisados nove artigos da síntese 
qualitativa desta revisão na literatura que apresentaram quais práticas são utilizadas, associado ao tratamento medicamentoso do câncer infantil. Observa-se uma distribuição temporal estável na divulgação dos artigos, nos últimos cinco anos. A maioria dos artigos foram publicados em língua inglesa.

A Tabela 1 apresenta os resultados obtidos segundo a temática que abordaram, informações acerca dos estudos realizados, como número do artigo, ano da publicação, título do artigo, autores, título do periódico, objetivos, amostra e resultado.

Tabela 1 - Apresentação dos artigos que integram a Revisão Integrativa.

\begin{tabular}{|c|c|c|c|c|c|c|}
\hline $\mathbf{N}$ & Data & Título & Autores & Periódico & Objetivos & Resultados \\
\hline 01 & 2020 & $\begin{array}{l}\text { Mothers' Attitudes } \\
\text { toward the Use of } \\
\text { Complementary and } \\
\text { Alternative } \\
\text { Medicine in } \\
\text { Children with } \\
\text { Cancer }\end{array}$ & $\begin{array}{c}\text { Dadgari et } \\
\text { al }\end{array}$ & $\begin{array}{l}\text { International } \\
\text { Journal of } \\
\text { Cancer } \\
\text { Management }\end{array}$ & $\begin{array}{l}\text { Determinar as } \\
\text { atitudes das mães } \\
\text { em relação ao uso } \\
\text { da medicina } \\
\text { complementar e } \\
\text { alternativa em } \\
\text { crianças com } \\
\text { câncer. }\end{array}$ & $\begin{array}{l}\text { No geral, 83,34\% das mães mencionaram orar } \\
\text { como uma forma de prática complementar e } \\
\text { alternativa para aumentar a chance de } \\
\text { recuperação da criança. } 74 \% \text { relataram } \\
\text { recuperação geral da criança, } 33,6 \% \text { evitaram } \\
\text { informar o médico sobre o uso de } \\
\text { medicamentos complementares e alternativos e } \\
76 \% \text { sustentaram que, devido à objeção dos } \\
\text { médicos, evitaram informá-lo. }\end{array}$ \\
\hline 02 & 2019 & $\begin{array}{l}\text { The Power of Reiki: } \\
\text { Feasibility and } \\
\text { Efficacy of } \\
\text { Reducing Pain in } \\
\text { Children with } \\
\text { Cancer Undergoing } \\
\text { Hematopoietic Stem } \\
\text { Cell } \\
\text { Transplantation }\end{array}$ & $\begin{array}{l}\text { Zucchetti } \\
\text { et al }\end{array}$ & $\begin{array}{l}\text { Journal } \\
\text { Pediatric } \\
\text { Oncology } \\
\text { Nursing }\end{array}$ & $\begin{array}{l}\text { Testar a } \\
\text { viabilidade e } \\
\text { eficácia do Reiki } \\
\text { para fornecer } \\
\text { alívio da dor entre } \\
\text { pacientes } \\
\text { pediátricos } \\
\text { submetidos ao } \\
\text { transplante de } \\
\text { células-tronco } \\
\text { hematopoiéticas } \\
\text { (TCTH). }\end{array}$ & $\begin{array}{l}\text { O efeito de } 88 \text { sessões de terapia Reiki em } \\
\text { nove pacientes (média de idade }=12 \text { anos; } \\
\text { Feminino }=61 \% \text { ) foi analisado sob uma } \\
\text { perspectiva de curto, médio e longo prazo. A } \\
\text { diminuição da dor ocorreu no período } \\
\text { experimental em curto e médio prazo, } \\
\text { enquanto no período de acompanhamento, o } \\
\text { nível de dor permaneceu estável. }\end{array}$ \\
\hline 03 & 2019 & $\begin{array}{l}\text { Medical marijuana } \\
\text { uses for pediatric } \\
\text { oncology patients: } \\
\text { single institution } \\
\text { experience }\end{array}$ & Ofir et al & $\begin{array}{c}\text { Hematologia e } \\
\text { Oncologia } \\
\text { Pediátrica }\end{array}$ & $\begin{array}{l}\text { Alívio dos efeitos } \\
\text { colaterais do } \\
\text { tratamento } \\
\text { anticâncer como } \\
\text { parte integrante } \\
\text { dos cuidados } \\
\text { paliativos e de } \\
\text { suporte de } \\
\text { crianças com } \\
\text { câncer. }\end{array}$ & $\begin{array}{l}\text { Os efeitos positivos foram relatados por } \\
\text { crianças verbais e pais em } 80 \% \text { dos casos. O } \\
\text { uso de maconha medicinal foi geralmente bem } \\
\text { tolerado com poucos pacientes relatando } \\
\text { toxicidade, com as reações adversas mais } \\
\text { comuns sendo queimação na garganta e } \\
\text { ataques de ansiedade em indivíduos que } \\
\text { optaram por fumar o produto. }\end{array}$ \\
\hline 04 & 2019 & $\begin{array}{l}\text { Utilisation des } \\
\text { médecines } \\
\text { complémentaires et } \\
\text { alternatives chez } \\
\text { l'enfant et } \\
\text { l'adolescent atteint } \\
\text { de cancer: une } \\
\text { pratique fréquente }\end{array}$ & $\begin{array}{c}\text { Menut et } \\
\text { al }\end{array}$ & $\begin{array}{l}\text { Bulletin du } \\
\text { Cancer }\end{array}$ &  & $\begin{array}{l}\text { Entre os } 202 \text { pacientes selecionados, } 111 \\
\text { famílias }(55 \%) \text { responderam. Cinquenta e } \\
\text { quatro }(48,6 \%) \text { usaram medicina } \\
\text { complementar e alternativa para seus filhos, } \\
\text { incluindo } 47(87 \%) \text { durante o tratamento inicial } \\
\text { do câncer. Trinta e dois }(59,3 \%) \text { falaram com } \\
\text { um profissional de saúde, incluindo } 25(75,8 \%) \\
\text { com seu oncologista. Os três medicamentos } \\
\text { complementares e alternativos mais utilizados } \\
\text { foram homeopatia (50\%), recurso a } \\
\text { magnetizador }(42,6 \%) \text { e osteopatia }(31,5 \%) \text {. A } \\
\text { principal motivação foi a redução dos efeitos } \\
\text { colaterais ao tratamento }(85,2 \%) \text {. A pontuação } \\
\text { média de satisfação percebida de } 7,4 / 10 \text {. }\end{array}$ \\
\hline 05 & 2018 & $\begin{array}{l}\text { amilienorientierte } \\
\text { Kunsttherapie für } \\
\text { Kinder und } \\
\text { Jugendliche mit } \\
\text { Krebs sowie } \\
\text { deren Eltern im } \\
\text { Akutkrankenhaus }\end{array}$ & Ortiz et al & $\begin{array}{l}\text { Psico- } \\
\text { oncologia }\end{array}$ & $\begin{array}{l}\text { Desenvolvimento } \\
\text { e teste de uma } \\
\text { intervenção } \\
\text { terapêutica } \\
\text { artística orientada } \\
\text { para a família. }\end{array}$ & $\begin{array}{l}\text { A oferta provou ser viável e, em princípio, } \\
\text { praticável na prática clínica diária. No entanto, } \\
\text { houve adversidades organizacionais na } \\
\text { implementação no que diz respeito à } \\
\text { abrangência estimada das unidades de } \\
\text { arteterapia, bem como na aquisição de } \\
\text { participantes. Os participantes afirmaram que a } \\
\text { intervenção ajudou a aliviar emocionalmente, }\end{array}$ \\
\hline
\end{tabular}




\begin{tabular}{|c|c|c|c|c|c|c|}
\hline $\mathbf{N}$ & Data & Título & Autores & Periódico & Objetivos & Resultados \\
\hline & & & & & & 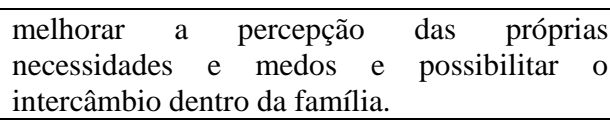 \\
\hline 06 & 2017 & $\begin{array}{l}\text { Beliefs and } \\
\text { determinants of the } \\
\text { use of traditional } \\
\text { complementary/ } \\
\text { alternative medicine } \\
\text { in pediatric patients } \\
\text { undergoing cancer } \\
\text { treatment in South } \\
\text { America }\end{array}$ & $\begin{array}{c}\text { Rocha et } \\
\text { al }\end{array}$ & $\begin{array}{l}\text { Journal of } \\
\text { Global } \\
\text { Oncology }\end{array}$ & $\begin{array}{l}\text { Investigar os } \\
\text { padrões, crenças e } \\
\text { determinantes do } \\
\text { uso de práticas } \\
\text { alternativas e } \\
\text { complementares } \\
\text { entre crianças sul- } \\
\text { americanas com } \\
\text { câncer. }\end{array}$ & $\begin{array}{l}\text { Verificamos que o uso de práticas alternativas } \\
\text { e complementares (PICS) era comum na } \\
\text { Argentina (47\%) e no Uruguai (76\%). } \\
\text { Variações nas formas de PICS utilizadas foram } \\
\text { observadas entre os países; no entanto, ambos } \\
\text { os países usaram PICS principalmente para } \\
\text { cuidados de suporte. Educação, índice de } \\
\text { riqueza e sistema de crenças foram preditores } \\
\text { significativos da escolha de PICS. }\end{array}$ \\
\hline 07 & 2016 & $\begin{array}{l}\text { Perspectives of } \\
\text { health professionals } \\
\text { and parents about } \\
\text { complementary } \\
\text { alternative medicine } \\
\text { in children with } \\
\text { cancer In Indonesia }\end{array}$ & $\begin{array}{c}\text { Susilawati } \\
\text { et al }\end{array}$ & $\begin{array}{l}\text { Asian Pacific } \\
\text { Journal of } \\
\text { Cancer } \\
\text { Prevention }\end{array}$ & $\begin{array}{l}\text { Este estudo } \\
\text { explorou } \\
\text { comparou as } \\
\text { perspectivas das } \\
\text { práticas } \\
\text { integrativas e } \\
\text { complementares } \\
\text { (PICS) de } \\
\text { profissionais de } \\
\text { saúde e pais de } \\
\text { pacientes jovens } \\
\text { com câncer na } \\
\text { Indonésia. }\end{array}$ & $\begin{array}{l}\text { Os pais eram mais propensos do que os } \\
\text { profissionais de saúde a pensar que a } \\
\text { quimioterapia pode curar o câncer }(80 \% \text { em } \\
\text { comparação com } 69 \%, \mathrm{P}=0,013) \text {. Quase } \\
\text { metade de todos os pais }(46 \%) \text { e profissionais } \\
\text { de saúde }(45 \%) \text { duvidaram que CAMcan } \\
\text { curasse o câncer. Os pais eram mais propensos } \\
\text { do que os profissionais de saúde a pensar que } \\
\text { as PICS pode ser útil no tratamento do câncer } \\
\text { infantil (54\% em comparação com } 35 \%, \mathrm{P}= \\
0,003) \text { A PICS mais recomendada pelos } \\
\text { profissionais de saúde foi a auto oração (93\%). } \\
\text { Os motivos para a indicação da PICS foram: } \\
\text { esperança de melhora do quadro da criança } \\
\text { (48\%), paciente deseja interromper o } \\
\text { tratamento (42\%). A maioria dos profissionais } \\
\text { de saúde desencorajados foram pessoas idosas } \\
\text { e inteligentes (70\%), as razões são: falta de } \\
\text { evidência de utilidade (77\%), falta de } \\
\text { conhecimento do CAM (75\%). }\end{array}$ \\
\hline 08 & 2016 & $\begin{array}{l}\text { Use of herbs or } \\
\text { vitamin / mineral / } \\
\text { nutrient } \\
\text { supplements by } \\
\text { cancer patients } \\
\text { pediatric }\end{array}$ & $\begin{array}{l}\text { Turhan \& } \\
\text { Bör }\end{array}$ & $\begin{array}{l}\text { Complementa } \\
\text { ry therapies in } \\
\text { clinical } \\
\text { practice }\end{array}$ & $\begin{array}{l}\text { Estudar a } \\
\text { frequência e os } \\
\text { fatores que afetam } \\
\text { o uso de medicina } \\
\text { complementar e } \\
\text { alternativa em } \\
\text { crianças } \\
\text { câncer. }\end{array}$ & $\begin{array}{l}\text { O tratamento com terapias integrativas e } \\
\text { complementares (PICS) mais comumente } \\
\text { usado foi o melaço de uva }(36,6 \%) \text {. A principal } \\
\text { fonte de informação para as famílias era a } \\
\text { internet. Nenhuma correlação encontrada entre } \\
\text { o uso de PICS e a escolaridade dos pais, o } \\
\text { nível de renda, o nível socioeconômico e o } \\
\text { tratamento quimioterápico. Pacientes com } \\
\text { câncer tendem a usar o tratamento PICS sem } \\
\text { informar os profissionais de saúde. }\end{array}$ \\
\hline 09 & 2016 & $\begin{array}{l}\text { The use of } \\
\text { alternative or } \\
\text { complementary } \\
\text { treatment in } \\
\text { pediatric cancer } \\
\text { patients: Survey of } \\
100 \text { cases in a level } \\
\text { III care institute }\end{array}$ & $\begin{array}{l}\text { Isaac- } \\
\text { Otero et al }\end{array}$ & $\begin{array}{l}\text { Gazeta } \\
\text { Médica do } \\
\text { México. }\end{array}$ & $\begin{array}{l}\text { Investigar a } \\
\text { frequência e as } \\
\text { causas do uso de } \\
\text { práticas } \\
\text { integrativas e } \\
\text { complementares } \\
\text { (PICS) } \\
\text { pacientes } \\
\text { oncológicos } \\
\text { pediátricos no } \\
\text { Instituto Nacional } \\
\text { de Pediatría } \\
\text { (INP). }\end{array}$ & $\begin{array}{l}\text { Cinquenta e um por cento dos cuidadores } \\
\text { entrevistados aceitaram o uso de algum tipo de } \\
\text { PICS; as terapias biológicas foram os } \\
\text { tratamentos mais utilizados e } 73 \% \text { ficaram } \\
\text { satisfeitos com os resultados. Os cuidadores } \\
\text { disseram aos seus médicos que estavam usando } \\
\text { o PICS em } 35 \% \text { dos questionários analisados, e } \\
\text { apenas } 2 \% \text { dos médicos perguntaram } \\
\text { diretamente sobre o seu uso. Nenhum dos } \\
\text { cuidadores substituiu ou interrompeu o } \\
\text { tratamento alopático. Análise: Esses } \\
\text { questionários revelam que mais da metade dos } \\
\text { cuidadores usam PICS. Acreditamos que as } \\
\text { crenças supersticiosas e culturais, bem como o } \\
\text { desejo dos cuidadores de participar ativamente } \\
\text { do tratamento do paciente, são as principais } \\
\text { causas do uso do PICS; no entanto, não } \\
\text { notificam seus médicos e isso pode afetar o } \\
\text { tratamento quimioterápico de maneiras ainda } \\
\text { não investigadas }\end{array}$ \\
\hline
\end{tabular}


de dados PUBMED, correspondendo quatro artigos, seguido de um na LILACS, três na WEB OF SCIENCE e um na SCOPUS. As nove publicações são de periódicos e estudos internacionais. Ainda, verificou-se que, quando especificadas as práticas integrativas e complementares, os estudos tiveram amostras diversas, como por exemplo, o uso do Reike, Apiterapia, Arteterapia, Plantas medicinais (fitoterapia) e Terapia comunitária integrativa.

A partir da análise obteve-se que, em relação aos objetivos, alguns estudos tiveram como foco central determinar as atitudes das mães em relação ao uso da medicina complementar e alternativa em crianças com câncer, isso se deve ao fato que, ao relatar a associação das práticas integrativas e complementares, a avaliação e participação da família foi necessária para melhor interpretação dos resultados, uma vez que o público avaliado não poderia fornecer respostas amplamente concretas ou relatar visualmente melhoras no seu quadro clínico com os métodos associados devido à idade (Dadgari, Bagheri, Salmani, et al., 2020).

O estudo de Zucchetti, Candela, Bottigelli e colaboradores (2019), focou em uma única PIC, abordando o tratamento convencional do câncer pediátrico e a eficácia do Reiki para fornecer alívio da dor entre pacientes pediátricos submetidos ao transplante de células-tronco hematopoiéticas. Tal estudo analisou a aplicação dessa prática em curto, médio e longo prazo, e obteve como resultado final que a utilização do reiki se mostrou eficaz para diminuição da dor em curto e médio prazo, contudo o alívio não se manteve a longo prazo.

Ofir, Bar-Sela, Weyl Ben-Arush e colaboradores (2019), investigaram o alívio dos efeitos colaterais do tratamento anticâncer como parte integrante dos cuidados paliativos e de suporte de crianças com câncer, relatando toxicidade, com as reações adversas mais comuns sendo queimação na garganta e ataques de ansiedade em indivíduos que optaram por fumar o produto. relatados por crianças verbais e pais em $80 \%$ dos casos.

Outro estudo (Menut, Seigneur, Gras Leguen et al., 2019) avaliou a prevalência do uso de medicamentos complementares e alternativos em oncologia pediátrica e caracterizou suas modalidades de uso e dos efeitos colaterais do tratamento anticâncer como parte integrante dos cuidados paliativos e de suporte de crianças com câncer, os três medicamentos complementares e alternativos mais utilizados foram homeopatia (50\%), recurso a magnetizador $(42,6 \%)$ e osteopatia $(31,5 \%)$.

Ortiz, Buttstädt, Richter e colaboradores (2019), desenvolveram um teste de uma intervenção terapêutica artística orientada para a família. Os participantes afirmaram que a intervenção ajudou a aliviar emocionalmente, melhorar a percepção das próprias necessidades e medos e possibilitar o intercâmbio dentro da família.

Em relação ao uso das terapias complementares para a população infantil a família parece ser peça chave para um bom resultado das intervenções, segundo (Rocha, Ladas, Lin e colaboradores (2017), as mães foram consideradas ótimas fontes de riqueza de informação para ter resultados significativos das práticas complementares, principalmente no que tange os padrões e crenças de cada região e cultura regional.

Para Susilawati, Sitaresmi, Handayani e colaboradores (2016), os pais são mais propensos a aceitarem e acreditarem nessas alternativas para a cura dos filhos, e a auto-oração foi o recurso mais aceitavél e utilizado entre o grupo amostral, revelando resultados significativos de evolução e principalmente a identificação da esperança na melhora do quadro da criança.

Turhan e Bör (2016) estudaram a frequência e os fatores que afetam o uso de medicina complementar e alternativa em crianças com câncer. Relataram que a principal fonte de informação para as famílias era a internet, que muitos pacientes com câncer tendem a usar diversos recursos sem informar aos profissionais de saúde, identificando o uso do melaço de uva em $36,6 \%$ da sua amostra como objetivo dos pais em melhorar o quadro patológico das crianças em tratamento, pois obtiveram informações que a ingestão da uva poderia ajudar no combate ao câncer e reduzir o crescimento de tumores.

Isaac-Otero, Molina-Alonso, Asencio-López e colaboradores (2016), revelam que os cuidadores entrevistados, em sua maioria, aceitam o uso de algum tipo prática complementar e alternativa, mas que as terapias biológicas foram os tratamentos mais utilizados pelos pacientes do seu grupo amostral, os quais ficaram satisfeitos com os resultados. Além disso, os 
cuidadores disseram aos seus médicos que estavam usando o ACT em 35\% dos questionários analisados, e apenas $2 \%$ dos médicos perguntaram diretamente sobre o seu uso. Os autores referem que as crenças supersticiosas e culturais, bem como o desejo dos pais de participar ativamente do tratamento do paciente, são as principais causas do uso das práticas complementares e alternativas.

Os cânceres identificados nos estudos tratam de pacientes pediátricos submetidos ao transplante de células-tronco hematopoiéticas. Alguns estudos não apresentaram o tipo de câncer, mas sim o relato da doença já instalada e como foi feita a associação das PICS ao tratamento e à companhia de familiares. As modalidades das práticas integrativas e alternativas são vastas, porém das 29 registradas no SUS, nem todas foram identificadas.

\section{Discussão}

A saber, a arteterapia é uma prática reconhecida e bastante utilizada para crianças e adolescentes com câncer, pois esse público são afetados pelas consequências psicológicas, assim como os cuidadores que passam por esse omento à beira do leito, em seu estudo Ortiz e colaboradores 2018 relatam que, os participantes que passaram pela intervenção com essa prática mostraram melhoras e foi algo significativo que ajudou aliviar seu quadro emocional, bem como a percepção das próprias necessidade e medos. Não só isso, mas utilizar a arteterapia durante o tratamento pode fornecer uma percepção mais sofisticada e uma reflexão pessoal dentro da família desses pacientes, claro, quando analisada e supervisionada por um profissional capacitado e experiente nessa avaliação.

Dito isso, podemos compreender que o uso de medicina complementar e alternativa é algo comum entre as mães, pois as mesmas são fontes de informações consideradas adequadas para responder e complementar o caso clínico com informações aos oncologistas. Segundo Dadgari e colaboradores em 2020, as atitudes das mães em relação ao uso de medicina complementar e alternativa em crianças com câncer no geral é relatado como tentativa de aumentar as chances de recuperação de seus filhos, que em alguns momentos, determinados pais aplicam essas práticas sem apoio ou suporte de profissionais específicos para tal alternativa.

Além disso, estudos demonstram a viabilidade do uso da terapia complementar em pacientes pediátricos com câncer, como por exemplo o uso do Reike. Não só isso, mas o estudo proposto por Zucchetti e colaboradores em 2019 revela que enfermeiras treinadas em oncologia pediátrica podem inserir o Reiki em sua prática clínica como um instrumento válido para diminuir o sofrimento causado pelo câncer infantil.

A saber, a maconha medicina é considerado um fitoterápico que pode servir como uma terapia complementar potencialmente útil ao tratamento de suporte convencional de crianças que sofrem de câncer, todavia, a utilização dessa prática requer preparo e administração correta sobre o controle de doses e prescrição, acompanhado de avaliações e diagnósticos feitos por profissionais capacitados para tal opção de complemento durante o tratamento.

Desse modo, é possível observar que a prevalência de administração de medicamentos complementares e alternativos é alta. Menut e colaboradores (2019) reforçam que são necessárias pesquisas para melhorar a comunicação e a qualidade do conselho à família, bem como mecanismos de ação e segurança da medicina alternativa, pois a utilização dessas práticas é prevalente em pacientes infantis com câncer.

Não só isso, mas o uso de ervas ou suplementos de vitaminas por pacientes oncológicos pediátricos é bastante comum. Para Turhan e Bör em 2016, o uso da medicina complementar e alternativa é crescente, e em seu estudo busca a frequência que afetam seu uso em crianças com câncer. Os autores relatam que o tratamento medicinal mais comumente usado por pais foi o melaço de uva, pois a fonte principal de informação para as famílias era a internet, e tais famílias tendem a usar o melaço para complemento do tratamento sem informar os profissionais de saúde.

Contudo, a gama de opções de utilização das práticas integrativas para o público infantil, em diversos países são 
utilizadas e por muitas vezes de formas diferentes, tais práticas comumente conhecidas podem ter correlação ao passado, quando comparados aos curandeiros, que são pessoas que utilizavam ervas e outros recursos como o toque que se assimila ao reiki entre outras características práticas na tentativa de curar ou amenizar o quadro de dor de crianças com algum tipo de câncer.

Para Rocha e colaboradores em 2017, as práticas integrativas e a medicina alternativa tem sido bastante documentadas, porém tão pouco se sabe sobre o uso delas em crianças com câncer. Isso se deve ao fato que, as práticas integrativas variam de forma de aplicação em vários países, que por questões culturais muitos familiares buscam esse recurso como suporte para aliviar o sofrimento das crianças, principalmente onde o acesso a medicamentos é limitado.

Não só isso, mas avaliar as perspectivas de profissionais de saúde e pais sobre a medicina alternativa em crianças com câncer também se faz necessário, com essa proposta de análise Susilawati e colaboradores em 2016 em seu estudo comprova que, quase metade de todos os pais e profissionais duvidaram que a medicina alternativa curasse o câncer, ainda assim, os pais foram mais propensos do que os profissionais a pensar que o uso de medicamentos alternativos e complementares fosse mais útil no tratamento do câncer infantil. Todavia, o recurso mais recomendado pelos profissionais foi a auto-oração, tais motivos para a indicação se deve ao fato que, aumentava a esperança de melhora do quadro da criança.

Para Isaac-Otero em 2016, o uso da medicina complementar é entendido como o uso de substâncias destinadas a coadjuvar com medicamentos complementares, sem tirar o efeito ou atrapalhar a sistematização da medicação central focada ao tratamento específico de cada criança, enquanto a medicina alternativa é considerada uma prática a curar o doente por si mesma, sendo utilizada de forma independente.

Não só isso, mas seu estudo relata ainda que, os cuidadores disseram aos seus médicos que estavam usando algum tipo de tratamento alternativo em $35 \%$ dos questionários analisados, e apenas $2 \%$ dos médicos perguntaram diretamente sobre o seu uso. Isso se deve ao fato que, esses questionários revelam que mais da metade dos cuidadores usam as práticas alternativas devido as crenças supersticiosas e culturais.

Sendo assim, ao se tratar do câncer infantil, podemos identificar tal patologia em diferentes formas e evoluções, segmentadas por avaliações, prescrições e opções de tratamento, utilizando a radioterapia, quimioterapia ou os dois associados dependendo do nível patológico da doença já instalada e identificada. De forma geral, independentemente do tipo de câncer as práticas integrativas e complementares se mostram eficazes quando associadas ao tratamento.

\section{Conclusão}

Conclui-se que todos os estudos apresentados apontam uma contribuição significativa das práticas integrativas associadas ao tratamento do câncer infantil e pode se mostrar forte aliada, gerando benefícios não apenas ao bem-estar, evolução no quadro de tratamento de certos pacientes, mas, à família dessa população que, por muitas vezes, também sofre com o período de tratamento.

Quando pensamos em práticas integrativas, logo imaginamos o paciente em si como foco principal, porém, se tratando de crianças, a família por sua vez se faz presente durante o tratamento oncológico e consequentemente precisam e podem participar da prática integrativa para ter um melhor resultado, assim, basicamente cuidar de quem cuida, os profissionais da saúde podem capacitar e orientar as famílias a utilizarem as práticas para realizarem o autocuidado.

A grande parte dos estudos especificamente voltados para crianças, atualmente advêm de pesquisas internacionais, o que fornece um ponto de partida útil para desenvolver uma compreensão não apenas aos benefícios, mas formas de que abrange a divulgação e popularização dessa metodologia associada a prática para profissionais brasileiros, abraçando o campo da pesquisa e atuação clínica.

Assim, sugere-se replicar esses achados em outros estudos mais aprofundados. Além disso, existe uma lacuna sobre 
outros tipos de práticas integrativas e complementares, não citadas nesse estudo, para desenvolver uma compreensão mais abrangente sobre o tema; além de estudos que relatem a aplicabilidade de certas práticas para melhor atuação do profissional que pretende trabalhar com PICS.

\section{Referências}

Alves, K. Y. A., Nascimento, C. P. A. do, Assis, Y. M. S. de, Salvador, P. T. C. de O., Tourinho, F. S. V., \& Santos, V. E. P. (2015). Integrative and complementary practices in oncologic treatment and the role of nursing. Revista de Pesquisa Cuidado é Fundamental Online, 7(4), 3163-3174. https://doi.org/10.9789/2175-5361.2015.v7i4.3163-3174

Bardin, L. (2016). Análise de Conteúdo. Editora 70.

Brasil. Departamento de Atenção Básica, Secretaria de Atenção à Saúde, Ministério da Saúde. (2015) Política Nacional de Práticas Integrativas e Complementares no SUS: atitude de ampliação de acesso. $2^{\text {a }}$ Ed. Brasília: Ministério da Saúde. 98

Caires, J. S., Andrade, T. A., Amaral, J. B., Calasans, M. T. de A., \& Rocha, M. D. S. (2014). A Utilização Das Terapias Complementares Nos Cuidados Paliativos: Benefícios E Finalidades. Cogitare Enfermagem, 19(3), Article 3. https://doi.org/10.5380/ce.v19i3.33861

Costa, A. R., Silva, R. S., Feitosa, R. M., Oliveira, K. K., \& Coelho, W. A. (2020). Práticas integrativas e complementares em saúde no cotidiano de crianças com câncer. Revista Enfermagem Atual In Derme, 92(30). https://doi.org/10.31011/reaid-2020-v.92-n.30-art.602

Dacal, M. del P. O., \& Silva, I. S. (2018). Impactos das práticas integrativas e complementares na saúde de pacientes crônicos. Saúde em Debate, 42, 724-735. https://doi.org/10.1590/0103-1104201811815

Dadgari, A., Bagheri, I., Salmani, N., \& Mirakhor, M. (2020). Mothers' Attitudes toward the Use of Complementary and Alternative Medicine in Children with Cancer. International Journal of Cancer Management, 13(10), Article 10. https://doi.org/10.5812/ijcm.104870

Habimorad, P. H. L., Catarucci, F. M., Bruno, V. H. T., Silva, I. B. da, Fernandes, V. C., Demarzo, M. M. P., Spagnuolo, R. S., \& Patricio, K. P. (2020). Potencialidades e fragilidades de implantação da Política Nacional de Práticas Integrativas e Complementares. Ciência \& Saúde Coletiva, 25, 395-405. https://doi.org/10.1590/1413-81232020252.11332018

Isaac-Otero, G., Molina-Alonso, D., Asencio-López, L., \& Leal-Leal, C. (2016). The use of alternative or complementary treatment in pediatric oncologic patients: Survey of 100 cases in a level III attention institute. Gaceta Médica de México., 152, 5-9.

Marcião, L. G. de A., Sousa, V. N. F. de, Lima, P. E., Lima, L. H. C., Marcião, E. F., Alves, E. L. C., Lira, J. V. M., Moura, L. F. de, Olivetto, M. M. S., Silva, G. da, Ferreira, O. P. C., Rodrigues, A. G., Alves, C. P., Viana, J. B., \& Alencar, I. de. (2021). A importância da atenção Fisioterapêutica nos cuidados paliativos em pacientes com câncer. Research, Society and Development, 10(6), e46310616042-e46310616042. https://doi.org/10.33448/rsd-v10i6.16042

Menin, S. P. (2020). Benefícios no tratamento do câncer atrelado ao uso das Práticas Integrativas e Complementares. Revista Perspectiva: Ciência e Saúde, 5(1), Article 1. http://sys.facos.edu.br/ojs/index.php/perspectiva/article/view/411

Menut, V., Seigneur, E., Gras Leguen, C., Orbach, D., \& Thebaud, E. (2019). Utilisation des médecines complémentaires et alternatives chez l'enfant et l'adolescent atteint de cancer: Une pratique fréquente. Bulletin du Cancer, 106(3), 189-200. https://doi.org/10.1016/j.bulcan.2018.11.017

Miotto, P. (2018). Práticas integrativas e complementares na atenção primária: efeitos sobre os sintomas da síndrome climatérica. 2018. Trabalho de conclusão de curso (Residência Multiprofissional em Saúde da Família) - Repositório Institucional da UFSC: Universidade Federal de Santa Catarina. Florianópolis, SC, ed. 1, 26 abr. 2018 .

Ofir, R., Bar-Sela, G., Weyl Ben-Arush, M., \& Postovsky, S. (2019). Medical marijuana use for pediatric oncology patients: Single institution experience. Pediatric Hematology and Oncology, 36(5), 255-266. https://doi.org/10.1080/08880018.2019.1630537

Ortiz, J., Buttstädt, M., Richter, D., Schepper, F., \& Singer, S. (2019). Familienorientierte Kunsttherapie für Kinder und Jugendliche mit Krebs sowie deren Eltern im Akutkrankenhaus. Der Onkologe, 25(6), 529-539. https://doi.org/10.1007/s00761-018-0491-z

Rocha, V., Ladas, E. J., Lin, M., Cacciavillano, W., Ginn, E., Kelly, K. M., Chantada, G., \& Castillo, L. (2017). Beliefs and Determinants of Use of Traditional Complementary/Alternative Medicine in Pediatric Patients Who Undergo Treatment for Cancer in South America. Journal of Global Oncology, 10 .

Sales, E. M. P., Santos, J. K. M., Barbosa, T. B., \& Santos, A. P. dos. (2020). Fisioterapia, funcionalidade e COVID-19: revisão integrativa. Cadernos ESP Revista Científica da Escola de Saúde Pública do Ceará, 14(1), 68-73. //cadernos.esp.ce.gov.br/index.php/cadernos/article/view/368

Silva, G., Sousa, I., Cabral, M., Bezerra, A., \& Guimarães, M. (2020). Política Nacional de Práticas Integrativas e Complementares: Trajetória e desafios em 30 anos do SUS. Physis: Revista de Saúde Coletiva, 30. https://doi.org/10.1590/s0103-73312020300110

Souza, M. T. de, Silva, M. D. da, \& Carvalho, R. de. (2010). Revisão integrativa: O que é e como fazer. einstein (São Paulo), 8(1), 102-106. https://doi.org/10.1590/s1679-45082010rw1134

Susilawati, D., Sitaresmi, M., Handayani, K., Ven, P. van de, Sutaryo, null, Kaspers, G., \& Mostert, S. (2016). HealthCare Providers' and Parents' Perspectives on Complementary Alternative Medicine in Children with Cancer in Indonesia. Asian Pacific Journal of Cancer Prevention: APJCP, 17(7), 3235-3242.

Tesser, C., Sousa, I., \& Nascimento, M. (2018). Práticas Integrativas e Complementares na Atenção Primária à Saúde brasileira. Saúde em Debate, 42, 174188. https://doi.org/10.1590/0103-11042018s112 
Research, Society and Development, v. 10, n. 13, e512101321487, 2021

(CC BY 4.0) | ISSN 2525-3409 | DOI: http://dx.doi.org/10.33448/rsd-v10i13.21487

Turhan, A. B., \& Bör, Ö. (2016). Use of herbs or vitamin/mineral/nutrient supplements by pediatric oncology patients. Complementary Therapies in Clinical Practice, 23, 69-74. https://doi.org/10.1016/j.ctcp.2016.03.009

Ursi, E. S., \& Gavão, C. M. (2006). Prevenção de lesões de pele no perioperatório: Revisão integrativa da literatura. Revista Latino-Americana de Enfermagem, 14, 124-131. https://doi.org/10.1590/S0104-11692006000100017

Zucchetti, G., Candela, F., Bottigelli, C., Campione, G., Parrinello, A., Piu, P., Vassallo, E., \& Fagioli, F. (2019). The Power of Reiki: Feasibility and Efficacy of Reducing Pain in Children With Cancer Undergoing Hematopoietic Stem Cell Transplantation. Journal of Pediatric Oncology Nursing: Official Journal of the Association of Pediatric Oncology Nurses, 36(5), 361-368. https://doi.org/10.1177/1043454219845879 